\title{
Electronic contribution to the energetics of helically wrapped nanotubes
}

\author{
Andrew Wall and Mauro S. Ferreira \\ School of Physics, Trinity College Dublin, Dublin 2, Ireland \\ (Received 8 May 2006; revised manuscript received 16 November 2006; published 11 December 2006)

\begin{abstract}
Motivated by evidence of helical wrapping of polymeric molecules around nanotubes, we calculate the electronic contribution to the energetics of linear charge distributions around a nanoscale cylindrical structure. This contribution alone leads to an angular dependence on the total energy, suggesting the occurrence of preferential wrapping directions. We argue that this contribution may play a role in determining the wrapping geometry of continuous linear charge distributions surrounding a nanotube.
\end{abstract}

DOI: 10.1103/PhysRevB.74.233401

PACS number(s): 73.22.Dj, 73.43.Cd

Nanotubes are one example of low-dimensional nanoscopic structures that have been at the forefront of intensive scientific investigation for more than a decade. In addition to their remarkable intrinsic physical properties and enormous potential for technological applications, doped nanotubes have also been in focus due to the possibility of engineering desirable characteristics in nanotube-based devices.

Among a plethora of possible doping agents, charged onedimensional-like structures such as polymers and DNA molecules are known to interact with nanotubes and to produce unusual physical properties: The former affecting the mechanical, ${ }^{1}$ thermal, ${ }^{2,3}$ and electronic ${ }^{4}$ properties of nanotube-polymer composite materials and the latter being used as a technique to separate nanotubes of different nature..$^{5}$ As a result of this interaction, these molecules can sometimes coat the walls of a nanotube. One common feature observed in the coating morphology is the fact that the molecules tend to wrap around the tubular structures in a helical fashion. ${ }^{6,7}$ Despite previous suggestions that this helicity should follow the chirality of the nanotube, ${ }^{8}$ there are indications that these molecules are not capable of resolving the atomic structure of the underlying lattice. ${ }^{9}$ Therefore, any physical mechanism that favors chiral order without depending on the underlying atomic structure is a possible candidate to explain the helical wrapping of molecules. It is our goal to show that the electronic contribution to the total energy is one such mechanism.

Whereas the DNA and some of the polymer molecules may have many differences, they share the common characteristic of being classified as quasilinear charge distributions. Motivated by this commonality, we investigate the energetics of helically ordered one-dimensional-like charge distributions around nanotubes. It is worth emphasizing that this geometry results from the favorable balance between the entropic, elastic, and electronic contributions to the free energy of the system. ${ }^{9,10}$ On the assumption that the latter two are the dominant contributions to the energy balance, ${ }^{9}$ especially at low temperatures, we are particularly interested in the electronic part of the total energy. Here we show that this contribution leads to energetically-favorable wrapping directions that result from interference patterns between different sections of a wrapping strand. Rather than providing an exact quantitative description for the full energetic balance, in this paper we focus on simple models for the electronic structure of both the cylinder and the linear charge distribution with the purpose of highlighting qualitative features that must also appear in more quantitatively accurate methods.

We describe the nanotube as a two-dimensional electron gas wrapped around itself onto a cylinder of radius $R$. Because the presence of a charge distribution near the tube is expected to polarize the electron gas in its proximity, we include a helically symmetric potential to reproduce this effect. The electron gas is efficient at screening any extra charge appearing in the system, which suggests that this potential must be short ranged. For the sake of simplicity, we assume it to be a delta function. This allows a simplified approach in which all the key quantities are calculated analytically. Efficient screening is not an essential assumption and can be relaxed by assuming a longer-range potential with no qualitative difference to the conclusions here obtained. With these simplifications in place, we map the problem of helical charge distributions surrounding a nanotube as a cylindrical electron gas under the action of a helicallysymmetric delta-function potential. Figure 1(a) illustrates the path followed by the helical potential in three dimensions, also depicted by its unwrapped version in Fig. 1(b). By calculating the energetics of this system we hope to identify preferential wrapping angles that minimize the total electronic energy of the system.

There is a striking similarity between the unwrapped representation of Fig. 1(b) and the Kronig-Penney model, in which electrons experience a periodic potential formed by equidistant delta functions. In that model the total energy depends nonmonotonically on the scatterers separation, which suggests the existence of preferential spacings. The (a)
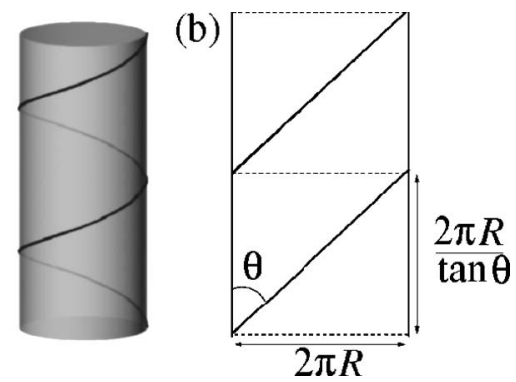

FIG. 1. Schematic diagram of the geometry involved in helical wrapping. (a) A single strand is assumed to wind around a cylinder of radius $R$ at an angle $\theta$. (b) In the two-dimensional depiction, the unwrapped tube is represented by a stripe of width $2 \pi R$ and the coiling angle $\theta$ defines a unit cell of length $2 \pi R / \tan \theta$. 
basic idea of the present paper is to explore this similarity and show that the total electronic energy of the helical charge distribution displays similar nonmonotonicities as a function of the separation $D$. Since $D$ is directly dependent on the angle $\theta$, the occurrence of optimal separation values may indicate the existence of preferential wrapping angles. In what follows we put these basic ideas to the test by calculating the total electronic energy of equally spaced delta functions, first in one dimension for illustration purposes, followed by its two-dimensional counterpart. After displaying the nonmonotonicity of the total energy in these illustrative cases, we include the appropriate boundary conditions that represent the helically wrapped tube and show that such a nonmonotonic behavior still persists in the wrapping geometry.

It is instructive to start with a one-dimensional electron gas in the presence of a single delta function $V(x)=\lambda \delta(x$ $-x_{0}$ ), which is a well-known textbook problem. ${ }^{11}$ Rather than adopting the usual method of solving the Schrödinger equation through the diagonalization of the Hamiltonian, we use the convenient method of expressing total energy variations through Green functions (GF). In this case the effect of the delta function on the total density of states of the electron gas is fully accounted for by a sum rule, also known as Lloyd's formula, ${ }^{12}$

$$
\Delta \rho(E)=-\frac{1}{\pi} \frac{d}{d E} \operatorname{Im} \ln \left(1-\lambda\left\langle x_{0}|\hat{g}| x_{0}\right\rangle\right) .
$$

$\Delta \rho(E)$ is the variation in the total density of states $\rho$ for electrons with energy $E, \hat{g}(E)=(E \hat{1}-\hat{H})^{-1}$ is the GF operator associated with the free-electron Hamiltonian $\hat{H}$, and the quantum basis $|x\rangle$ labels the eigenvectors of the position operator. A simple integration by parts provides the total energy variation $\delta \epsilon_{1}$ due to the introduction of a single delta function. It is given by

$$
\delta \epsilon_{1}=\frac{1}{\pi} \int_{-\infty}^{E_{F}} d E \operatorname{Im} \ln \left(1-\lambda\left\langle x_{0}|\hat{g}| x_{0}\right\rangle\right) .
$$

In the basis $|x\rangle$ the GF matrix element appearing in Eqs. (1) and (2) is given by

$$
\left\langle x_{0}|\hat{g}| x_{0}\right\rangle=-i \sqrt{\frac{1}{2\left(E+i 0^{+}\right)}},
$$

where $0^{+}$stands for a small imaginary part added to the energy $E$. Atomic units will be used throughout. The quantity $E_{F}$ is the Fermi level and regulates the electronic occupation of the system. As energy reference we choose the total energy of the perturbation-free electron gas, which means that the total energy of the system with a single perturbation is given by $\delta \epsilon_{1}$. The quantity $\lambda$, which is the weight of the associated delta function potential, acts as a parameter that fully determines the energy $\delta \epsilon_{1}$. We can then study how the total energy of a system composed of equally spaced localized perturbing potentials depends on their separation.

We consider an array of $N$ delta functions that are a distance $D$ apart. In this case the total energy of the system $\delta \epsilon_{N}(D)$ should not be an integer multiple of $\delta \epsilon_{1}$, i.e.,

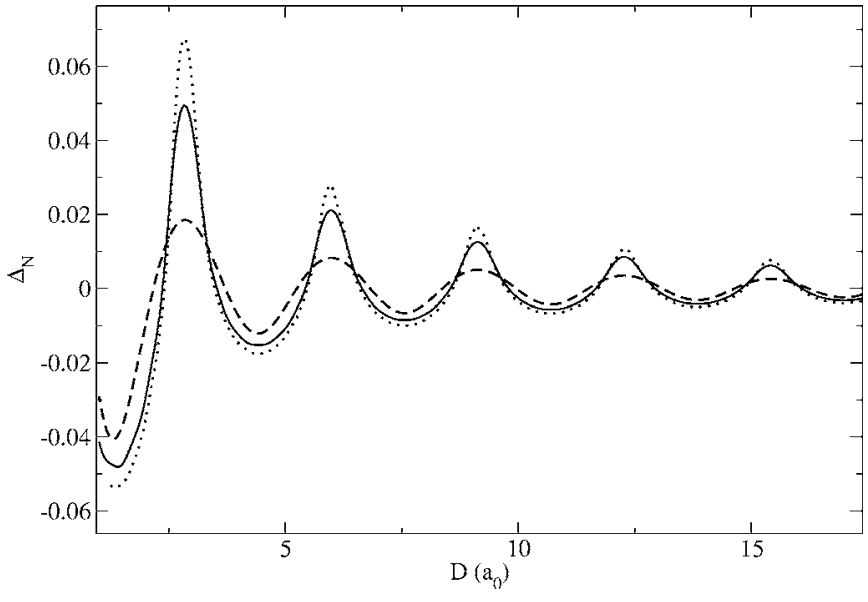

FIG. 2. Relative energy change per perturbation for a onedimensional free electron gas perturbed by a series of equally spaced delta-function scatterers for (i) two scatterers (dashed line); (ii) seven scatterers (continuous line); (iii) infinite number of scatterers (dotted line). The amplitude of the scattering potential is $\lambda$ $=-0.3$ in all cases. The distance between successive minima (maxima) is $\pi / k_{F}$, where $k_{F}$ is the Fermi wave number of the gas.

$\delta \epsilon_{N}(D) \neq N \delta \epsilon_{1}$. It is simple to generalize Eq. (2) to express the energy variation due to the introduction of $N$ perturbations. With the perturbing potential now described by $V(x)$ $=\lambda \sum_{j=1}^{N} \delta(x-j D)$, the total energy variation reads

$$
\delta \epsilon_{N}(D)=\frac{1}{\pi} \int_{-\infty}^{E_{F}} d E \operatorname{Im} \ln \operatorname{det}(\hat{1}-\lambda \hat{M})
$$

In the equation above, $\hat{1}$ is the identity matrix and $\hat{M}$ is a $N \times N$ matrix whose elements are $[M]_{\ell, m}=\langle\ell \times D|\hat{g}| m \times D\rangle$. We select $N \delta \epsilon_{1}$ as our energy unit and choose the dimensionless quantity $\Delta_{N}(D)$ defined as

$$
\Delta_{N}(D)=\frac{\delta \epsilon_{N}(D)-N \delta \epsilon_{1}}{N\left|\delta \epsilon_{1}\right|}
$$

to represent the separation dependence of the total energy. Figure 2 plots this quantity as a function of the spacing $D$ for different values of $N$. Also depicted is the case for $N=\infty$, which results from a simple Fourier transform from the quantum basis $|x\rangle$ to a super cell representation $\left|k_{x}, \chi\right\rangle$, where $k_{x}$ is a wave vector defined within the Brillouin zone $k_{x}$ $\in[-\pi / D, \pi / D]$ and $\chi$ is an intracell continuous variable defined within the range $\chi=[0, D)$. In this case, the expression for $\delta \epsilon_{\infty}$ becomes

$$
\begin{aligned}
\delta \epsilon_{\infty}(D)= & \frac{D}{2 \pi^{2}} \int_{-\pi / D}^{\pi / D} d k_{x} \int_{-\infty}^{E_{F}} d E[\operatorname{Im} \ln \operatorname{det}(1 \\
& \left.\left.-\lambda\left\langle k_{x}, 0|\hat{g}| k_{x}, 0\right\rangle\right)\right]
\end{aligned}
$$

where the GF matrix element in this new basis is given by 


$$
\left\langle k_{x}, 0|\hat{g}| k_{x}, 0\right\rangle=\left(\frac{e^{i k_{x} D}}{e^{i k_{x} D}-e^{i k_{0} D}}+\frac{e^{i k_{0} D}}{e^{-i k_{x} D}-e^{i k_{0} D}}\right)\left\langle x_{0}|\hat{g}| x_{0}\right\rangle,
$$

the GF matrix element $\left\langle x_{0}|\hat{g}| x_{0}\right\rangle$ was defined in Eq. (3), and $k_{0}(E)=\sqrt{2\left(E+i 0^{+}\right)}$. As anticipated, the total electronic energy depends nonmonotonically on the perturbation spacing $D$ and every single curve for $\Delta_{N}(D)$ shows a series of regularly spaced minima. This nonmonotonicity points to the existence of preferential separation values for which the total energy is minimized. The overall trend of damped oscillations corroborates the expected result that only in the asymptotic limit of infinitely large separation $D$ does the total energy $\delta \epsilon_{N}(D)$ agree with $N \delta \epsilon_{1}$.

We now extend the results derived above to deal with two-dimensional perturbations. As shown in Fig. 1, the presence of a helically symmetric delta function potential surrounding a nanotube can be represented in its unwrapped version by an array of equally spaced line delta functions spanning the finite-width stripe that one obtains from unwrapping the tube. To characterize a tube, this unwrapped representation must include periodic boundary conditions, which can be visualized through a simple juxtaposition of identical stripes depicted in Fig. 1(b). This generates a twodimensional array of infinitely long linear delta functions that can be mathematically expressed as $V(x, y)=\lambda \Sigma_{j} \delta(x$ $-j D)$. Adopting the same notation as before, the energy variation per unit length of perturbation due to a single line delta function located at $x=x_{0}$ becomes

$$
\delta \epsilon_{1}=\frac{1}{2 \pi^{2}} \int_{-\infty}^{\infty} d k_{y} \int_{-\infty}^{E_{F}} d E \operatorname{Im} \ln \left(1-\lambda\left\langle x_{0}, k_{y}|\hat{g}| x_{0}, k_{y}\right\rangle\right),
$$

where

$$
\left\langle x_{0}, k_{y}|\hat{g}| x_{0}, k_{y}\right\rangle=-i \sqrt{\frac{1}{2 E-k_{y}^{2} / 2}} .
$$

For the case of an infinite number of line delta functions located at equally spaced positions along the $x$ direction (separated by $D$ ), the total energy variation $\delta \epsilon_{\infty}$ is

$$
\begin{aligned}
\delta \epsilon_{\infty}= & \frac{D}{4 \pi^{3}} \operatorname{Im} \int_{-\pi / D}^{\pi / D} d k_{x} \int_{-\infty}^{\infty} d k_{y} \int_{-\infty}^{E_{F}} d E \ln (1 \\
& \left.-\lambda\left\langle k_{x}, 0, k_{y}|\hat{g}| k_{x}, 0, k_{y}\right\rangle\right)
\end{aligned}
$$

where the GF reads the same as Eq. (7), the only difference being that in this case the energy $E$ is replaced with $E$ $-k_{y}^{2} / 2$. The integration limits for the $k_{x}$ integral in Eq. (10) reflect the periodicity along the $x$ direction whereas those for the integral over $k_{y}$ confirm the free-electron character along that direction.

The definition of $\Delta_{N}(D)$ in Eq. (5) is still valid in this case and reflects how the total energy depends on the separation $D$ between two-dimensional line delta functions. Shown in Fig. 3(a) for $N=\infty$, the expected non-monotonicity of $\Delta_{\infty}(D)$ is confirmed, suggesting the existence of preferential values

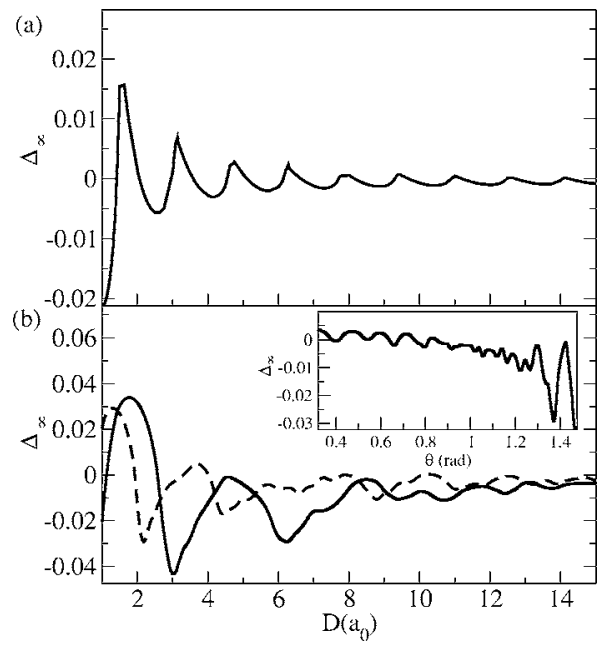

FIG. 3. Relative energy change per unit length of perturbation for (a) a two-dimensional free electron gas perturbed by an infinite array of equally spaced delta-function scatterers $\left(E_{F}=2.0\right)$ and (b) a free electron gas cylinder in the presence of a coiling perturbation for two separate Fermi levels $\left(E_{F}=0.5\right.$, solid line and $E_{F}=1.0$, dashed line). A series of energy minima is obtained, indicating the existence of energetically favorable coiling angles. Inset in (b) is a graph of $\Delta_{\infty}$ as a function of the coiling angle $\theta$. The amplitude of the scattering potential is $\lambda=-0.3$ in all cases. The distance between successive minima (maxima) is again seen to be $\pi / k_{F}$, where $k_{F}$ is the Fermi wave number of the gas.

for the spacing $D$. As previously mentioned, the existence of such preferential spacings may lead to the occurrence of ideal wrapping angles $\theta$, since the two quantities are directly related.

This speculation can only be tested if the appropriate boundary conditions for helically wrapped tubes are included. This is done by imposing the following quantization conditions for the vectors $k_{x}$ and $k_{y}$ :

$$
k_{y}=-\left(\frac{1}{\tan \theta}\right) k_{x}+\frac{m}{R \sin \theta},
$$

where $m$ is an integer. Rather than spanning an infinite-long stripe of finite width, the integration over $k_{x}$ and $k_{y}$ appearing in Eq. (10) must be performed under the constraint imposed by Eq. (11); that is, $k_{x}$ and $k_{y}$ vary over a set of discrete lines. Our speculation is finally confirmed after $\Delta_{N}(D)$ is evaluated with the aforementioned quantization constraints and still displays similar nonmonotonic behavior in the separationdependent total energy. Figure 3(b) plots $\Delta_{\infty}$ as a function of $D$ for a tube of radius $R=5 a_{0}$ and two arbitrary values of $E_{F}$. The periodicity of $\Delta_{\infty}(D)$ depends on the Fermi level but all curves display several minima at specific values of $D$. Following Fig. 1(b), the relation $D=2 \pi R \cos \theta$ means that preferential values for the spacing $D$ correspond to energetically favorable wrapping angles $\theta$. This is confirmed in the inset of Fig. 3 that shows how the total energy scales with the angle $\theta$.

As far as the electronic contribution to the total energy is concerned, the existence of preferential coiling angles does not determine the final wrapping direction of one- 
dimensional strands surrounding a nanotube. This must obviously be weighed against other energy contributions such as the elastic cost of folding the strands as well as other geometrical factors. ${ }^{9}$ Only the balance from all these contributions can determine the overall favorable alignment. In fact, according to Fig. 3(b), the optimal angle that minimizes the total electronic energy is close to $\theta=\pi / 2$. This wrapping angle would bring significant costs in terms of elastic energy. A compromise angle is likely to arise as a result of the adequate balance that accounts for the different contributions to the energy. Although the simplicity of our model is not capable of addressing the precise weights in this balance, it serves the purpose of pointing to this contribution as a possible mechanism that favors chiral order without depending on the underlying atomic structure.

Following the analogy to the Kronig-Penney model, it is simple to understand the origin of these total energy oscillations on a helically wrapped nanotube. The proximity of the continuous linear charge distribution polarizes the nanotube and induces a localized short-range potential that affects the electronic structure of the tube. These localized potentials produced by different sections of the wrapping strand generate an interference pattern that appears in the electronic wave functions of the underlying nanotube. Such a pattern is therefore responsible for the occurrence of the size-dependent oscillations of the total energy.

To test whether the nonmonotonicities of the total energy also appear in more realistic models, we have performed similar calculations within the tight-binding model. The presence of the continuous linear charge distribution is accounted for by once again assuming that it can polarize what lies in its close proximity. In this case, the polarizable entities are the carbon atoms on the tube. They acquire on-site potentials that differ from those associated with their nonpolarized counterparts. Similar to the electron gas case, we use Eq. (10) to write the energy variation due to the introduction of such a polarizing perturbation. Figure 4 shows the results for the tight-binding calculations of a $(9,0)$ zigzag nanotube

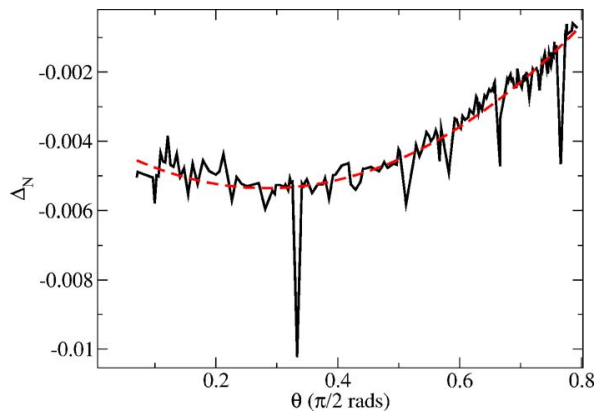

FIG. 4. (Color online) Angular dependence of the relative energy change $\Delta_{N}$ for helical wrapping on a $(9,0)$ zigzag nanotube. An overall nonmonotonic trend depicted by the dashed line is superimposed to short-period fluctuations that correspond to high-symmetry angles of the perturbation.

wrapped by a thin charge distribution that forms an angle $\theta$ with the tube axis. The nonmonotonicity in the angular dependence of the total energy can also be seen in this case, proving that it is not an exclusive feature of the electron gas model.

In summary, we have shown that the electronic contribution to the total energy of nanotubes wrapped by helical continuous charge distributions displays a nonmonotonic behavior as a function of the wrapping angle. The origin of these oscillations can be understood as a result of the formation of an interference pattern in the electronic wave functions. An analogy to the Kronig-Penney model was established to highlight the correlation between the oscillations and the electronic structure of the underlying nanotube. We argue that these oscillations may play a role in determining the preferential wrapping angles of helically symmetric charge distributions around nanotubes.

M.S.F. acknowledges the financial support of Science Foundation Ireland. A.W. acknowledges support received from the Irish Research Council for Science, Engineering and Technology under the EMBARK initiative.
${ }^{1}$ A. B. Dalton, S. Collins, E. Munoz, J. M. Razal, V. H. Ebron, J. P. Ferraris, J. N. Coleman, B. G. Kim, and R. H. Baughman, Nature (London) 423, 703 (2003).

${ }^{2}$ S. Berber, Y. K. Kwon, and D. Tománek, Phys. Rev. Lett. 84, 4613 (2000).

${ }^{3}$ P. Kim, L. Shi, A. Majumdar, and P. L. McEuen, Phys. Rev. Lett. 87, 215502 (2001).

${ }^{4}$ B. E. Kilbride, J. N. Coleman, J. Fraysee, P. Fournet, M. Cadek, A. Drury, S. Hutzler, S. Roth, and W. J. Blau, J. Appl. Phys. 92, 4024 (2002).

${ }^{5}$ M. Zheng, A. Jagota, E. D. Semke, B. A. Diner, R. S. Mclean, S. R. Lustig, R. E. Richardson, and N. G. Tassi, Nat. Mater. 2, 338 (2003).

${ }^{6}$ B. McCarthy, J. N. Coleman, S. A. Curran, A. B. Dalton, A. P.
Davey, Z. Konya, A. Fonseca, J. B. Nagy, and W. J. Blau, J. Mater. Sci. Lett. 19, 2239 (2000).

${ }^{7}$ M. J. O'Connell, P. Boul, L. M. Ericson, C. Huffman, Y. Wang, E. Haroz, C. Kuper, J. Tour, K. D. Ausman, and R. E. Smalley, Chem. Phys. Lett. 342, 265 (2001).

${ }^{8}$ R. Czerw, Z. Guo, P. Ajayan, Y.-P. Sun, and D. Carroll, Nano Lett. 1, 423 (2001).

${ }^{9}$ J. N. Coleman and M. S. Ferreira, Appl. Phys. Lett. 84, 798 (2004).

${ }^{10}$ A. Maritan, C. Micheletti, A. Trovato, and J. R. Banavar, Nature (London) 406, 287 (2000).

${ }^{11}$ E. Merzbacher, Quantum Mechanics (John Wiley \& Sons, Inc., New York, 1998), Chap. 6, pp. 107-108.

${ }^{12}$ R. Zeller, J. Phys.: Condens. Matter 16, 6453 (2004). 